\title{
Impact of Internalized Stigma on HIV Prevention Behaviors Among HIV-Infected Individuals Seeking HIV Care in Kenya
}

\author{
Caroline Kingori, Ph.D., M.P.H., ${ }^{1}$ Michael Reece, Ph.D., M.P.H. ${ }^{2}$ Samuel Obeng, Ph.D., ${ }^{3}$ \\ Maresa Murray, Ph.D., ${ }^{4}$ Enbal Shacham, Ph.D., M.Ed., M.P.E., ${ }^{5}$ Brian Dodge, Ph.D., ${ }^{2}$ \\ Emannuel Akach, MBChB, MMed, ${ }^{6}$ Peter Ngatia, Ph.D., and David Ojakaa, Ph.D. ${ }^{6}$
}

\begin{abstract}
In general, an initial diagnosis of HIV is likely to be correlated with the onset of HIV stigma. HIV-positive individuals are likely to internalize stigma, may suffer from psychosocial issues, or engage in maladaptive behaviors to cope with the diagnosis. Internalized stigma stems from fear of stigmatization also known as felt stigma. The current study examined the impact of HIV felt stigma on overall health and success of HIV prevention behaviors among 370 participants living with HIV and receiving care at an urban HIV clinic in Kenya. An 18-item instrument was cross culturally adapted to measure felt stigma. Descriptive and logistic regression analyses examined the data. Findings indicate that $25.9 \%(n=96)$ of participants who reported experiencing high levels of felt stigma related to other people's attitudes toward their condition, ostracizing, and a disruption of their personal life, were likely to not adhere to prescribed HIV medication and not disclose their HIV serostatus to one other person. Those who also experienced felt stigma related to a disruption of their personal lives while mediated by depression were likely to report poor overall health. Findings support having HIV clinics and interventions develop relevant HIV prevention strategies that focus on the emerging dimensions of felt stigma which can significantly impact disclosure of serostatus, medication adherence, and overall health.
\end{abstract}

\section{Introduction}

N sub-Saharan Africa, documented evidence reports a 1 decline in the number of new HIV infections from a peak of 2.2 million in 2001 to 1.9 million in 2010.$^{1,2}$ Nevertheless, South Africa reportedly bears the severity of the epidemic with 5.6 million people living with HIV as of 2010. ${ }^{3}$ In Kenya, there has been a decline in HIV incidence rates although HIV prevalence rates appear to have stabilized at high levels. ${ }^{4}$ For example, in Nyanza Province Western Kenya, reports from male circumcision programs indicate that almost 2000 new HIV infections were avoided by program participants in the year $2010 .^{1}$

A national AIDS strategic plan in 2006-2010 5 was launched to address HIV prevention challenges in Kenya such as negative serostatus partners in discordant relationships or neglected marginal groups at increased risk of acquiring HIV. ${ }^{6}$ To enhance the effectiveness of HIV prevention strategies, the Kenya National AIDS Control Council [NACC $]^{6}$ recommends involving political leaders and incorporating local culture and traditions such as including traditional midwives in prevention of mother-to-child transmission programs. The plan aimed at increasing universal access to HIV care, reducing burden of care in households and reducing AIDS mortality. ${ }^{5}$

Particular to stigma, an HIV stigma strategic plan was launched in 2008 in Kenya utilizing religious leaders alongside health care workers. Consequently, stigma has reportedly decreased via advocacy and social marketing messages. ${ }^{5}$ Such findings provide some hope but there is room for more work in order to eradicate HIV stigma in Kenya.

HIV-related stigma stems from personal beliefs or situational cues implying that an individual has deviated from societal norms by acquiring HIV and should be treated differently; leads to labeling, ostracizing, discriminating, or stereotyping of people living with $\mathrm{HIV} / \mathrm{AIDS}$ (PLWHA) ${ }^{7-12}$ Given the discrediting nature of HIV/AIDS because it is a sexually transmitted disease or associated with certain group

\footnotetext{
${ }^{1}$ Department of Social and Public Health, Ohio University, Athens Ohio.

${ }^{2}$ Center for Sexual Health Promotion, ${ }^{3}$ African Studies Program, ${ }^{4}$ Department of Applied Health Science, School of Health and Physical Education, Indiana University, Bloomington, Indiana.

${ }^{5}$ Department of Behavioral Sciences and Health Education, School of Public Health, Saint Louis University, St. Louis, Missouri.

${ }^{6}$ African Medical Research Foundation (AMREF), Nairobi, Kenya.
} 
memberships such as commercial sex workers, PLWHA are likely to internalize stigma and may suffer from low self-esteem, depression, anxiety, unproductivity or engage in maladaptive behaviors to cope. $7,9,10,13,14$

Internalized stigma stems from fear of stigmatization, which is termed as felt stigma. ${ }^{15,16}$ Ultimately, felt stigma can negatively impact PLWHA's overall health and the success of HIV prevention behaviors such as condom use, adherence to prescribed medication regimen, and disclosure of serostatus to others. ${ }^{10,17-19}$

In order to minimize HIV felt stigma and enhance the uptake of aforementioned HIV prevention behaviors among PLWHA in Kenya, it is important to incorporate local culture and traditions in HIV prevention strategies as recommended by NACC. ${ }^{6}$ Inclusion of culture and tradition may provide insight into understanding contexts "within which these fears, blames and behaviors are expressed."20(p410)

Cultural contexts include a network of group affiliations associated with family, friends, religious institutions, workplace, government etc. Simmel ${ }^{21(\mathrm{p} 163)}$ could not have said it better when he suggested that, "... society arises from the individual and that the individual arises out of association." Thus, group perspective is necessary in addressing HIV stigma that emerges from shared values, beliefs, norms at the individual, familial and societal levels, e.g., women who are seropositive are deemed promiscuous and immoral and blamed for infecting the man. ${ }^{22,23}$ Attention should, therefore, shift to understanding the network of relationships between a PLWHA and the aforementioned groups within his cultural context instead of placing the burden of behavior change on the individual. ${ }^{24}$

In summary, addressing stigma, particularly felt stigma, in HIV prevention is important because of the documented evidence highlighting that stigmatized individuals may perceive themselves as outcasts in their communities, engage in maladaptive behaviors (alcohol abuse or unprotected sex) as a coping mechanism and be unproductive. ${ }^{7,9,10,13-15}$ Other PLWHA may suffer from mental health issues especially if stigmatized individual perceives negative actions by other individuals as legitimate. Consequently, such an individual may birth negative concepts about self-based on the negative attitudes of others which may negatively impact overall health status. ${ }^{9,10}$

The current study examined the impact of HIV felt stigma on the overall health and success of HIV prevention behaviors among PLWHA in an urban city in Kenya. An 18item instrument was cross culturally adapted and utilized to measure felt stigma. Seven items addressing culturally relevant beliefs, values, traditions, and attitudes likely to be perceived and internalized by PLWHA as a response to their being HIV positive ${ }^{20,25-27}$ were added to the original 11-item HIV felt stigma instrument. ${ }^{28}$ The original 11-item tool was also an enhanced version of a 13-item HIV stigma measure. $^{29}$

\section{Methods}

\section{Data collection}

Study protocols were approved by the Ethics \& Scientific Review Committee at the African Medical Research Foundation (AMREF) in Nairobi and the Institutional Review Board at Indiana University-Bloomington.
Study participants $(n=370)$ were recruited from a voluntary testing and counseling (VCT) clinic during routine clinic visits, over a period of $3 \frac{1}{2}$ weeks in 2011, in a resource-limited urban area in Kenya. Eligible participants were either male or female, living with HIV/AIDS, between the ages of 18-60 years old, were receiving HIV / AIDS related and other health services at the clinic, and were from a diverse ethnic and socioeconomic background.

Potential participants attending the VCT clinic were approached by the researcher at the waiting area as they waited to be seen by a medical officer. A designated medical officer was on hand every morning to identify for the researcher HIV-positive individuals, primarily those who had medical appointments at the clinic on a given day. After participants were screened for eligibility they were recruited, taken to a private room, and asked to complete a researcher-administered questionnaire in both English and Swahili, both of which are national languages in Kenya.

Participation was voluntary and participants provided consent for their responses to be used to inform research. The VCT clinic provides health services pertaining to: HIV infection testing and counseling, antiretroviral therapy (ART) medication, preventing mother-to-child transmission of HIV (PMTCT) services, tuberculosis prevention, and maternity among others.

\section{Measures}

Demographic, sexual behavior, and HIV-related variables. Participants completed measures addressing demographics (age, gender, education status, number of children at home, employment status, religion, tribal affiliation, income); HIV-related characteristics (disclosure of serostatus, duration of HIV serostatus, social support); and sexual behavior (condom use the most recent time they had sex and number of sexual partners in the past 3 months).

Medication adherence scale. A four-item scale was adapted from Morisky et al. ${ }^{30}$ and has a 5-point scale response options ranging from $1=$ never to $5=$ always. The scale is reported as a reliable and valid measure for diagnosing comprehension levels of medical information in order to address any misinformation or misbeliefs and examining behaviors related to adherence of prescribed medication. ${ }^{30}$

HIV felt stigma instrument. Felt stigma was measured utilizing a cross culturally adapted 18-item tool. Felt stigma symptoms were based on a 30-day period using five response items, $1=$ never to $5=$ always. This instrument has previously been used in HIV clinics the United States ${ }^{28}$ and western Kenya. $^{31-33}$

Prior to conducting analyses for this article, factor analyses of the felt stigma tool were conducted; four factors with a total of 10 items emerged with a $45 \%$ total explained variance. All factor loadings had a 0.50 cutoff point and did not cross-load.

Factor one, public attitudes, had three items loaded on it with item factor correlations ranging from 0.628 to 0.547 and had the greatest percentage variance $(31.01 \%)$. The three items measured the extent to which PLWHA were affected by public beliefs and attitudes toward their serostatus. Factor two, ostracize, had three item loadings with item factor correlations ranging from 0.784 to 0.604 and explained variance 
of $6.63 \%$. Items examined stigmatizing feelings related to avoidance, looked down upon, or treated differently.

Factor three, discrimination, with a $4.56 \%$ explained variance had two item loadings with item factor correlations between 0.842-0.734. Items examined felt stigma related to other people not touching PLWHA or eating food prepared by PLWHA. Factor four, personal life disrupted, also had two item loadings with item factor correlations between 0.612 0.516 and explained variance of $2.88 \%$. Items measured the extent to which PLWHA had experienced a disruption of their home and work life due to the fear of stigmatization at home or place of employment.

The overall felt stigma tool revealed a high internal consistency $(\alpha=0.864)$ while the emerging four factors also had high internal consistency with $\alpha$ coefficients ranging from 0.675 to 0.799 .

The Patient Health Questionnaire-9 (PHQ-9). PHQ-9 $9^{34,35}$ was utilized to measure depressive symptoms because stigma is a constellation of psychological distress symptoms, which may mediate the impact of HIV stigma on likelihood of engaging in HIV prevention behaviors. ${ }^{36}$ Depression is documented to have an association with a diagnosis of HIV infection and may increase PLWHA perception of HIV stigma. ${ }^{36-38}$ The 9-item tool measured depression symptoms experienced over a 2-week period. It is made up of a 4-point Likert scale ranging from $0=$ not at all to $3=$ nearly every day and summed up across 9 severity items (minimal, mild, moderate, moderately severe, severe) ranging from 0 to 27 . In this study, PHQ-9 had a satisfactory level of internal consistency $(\alpha=0.702)$. The instrument has been utilized among other HIV-positive individuals. ${ }^{31,32,34,35,39-42}$

General health rating. The general rating measure was adopted from the SF- $12^{43}$ instrument to measure overall health utilizing response scales ranging from "excellent" (high score of 1) to "poor" (low score of 5).

\section{Data analysis}

All data analyses were conducted using SPSS version 19.0 (SPSS Inc., Chicago, IL). ${ }^{44}$ Descriptive tests examined demographic and HIV-related characteristics stratified by gender. Logistic regression analyses were conducted where five categories of independent variables (four HIV felt stigma dimensions and depression) were entered to determine which of them would predict a negative impact on each dependent variable (medication adherence, disclosure of serostatus to one other person, condom use) and overall health. HIV felt stigma factor scores, depression and dependent variables were recoded into categorical binary response variables $(0,1)$ to enable the analyses.

Depression sum scores ranged from 0 to 27 across severity categories: minimal $=1$, mild $=2$, moderate $=3$, moderately severe $=4$, and severe $=5$. These categories were then collapsed and recoded into two binary categories (mild depression, 1 through $3=0$ and severe depression, 4 through $5=1$ ). Felt stigma sum scores were also collapsed and recoded into two binary categories whereby low levels included sum scores of 39 or less while high levels had sum scores of 40 or higher.

The general health rating response levels (excellent $=5$, very $\operatorname{good}=4$, $\operatorname{good}=3$, fair $=2$, poor $=1$ ) were collapsed into two binary categories: optimal, 5 through $3=0$ and poor, 2 through $1=1$.

\section{Results}

\section{Participant characteristics}

Data were analyzed for 370 participants. The sample had a mean age of 37.06 years (standard deviation $[S D]=8.61$ ), $51.1 \%$ were married, and $61.1 \%$ were female (Table 1 ). The highest level of education for most of the participants was "standard 4-8" (48.1\%) and "form 3-4" (24.3\%), which is equivalent to the American system of middle school grades 6-8 and high school grades 9-12. Due to the nature and location of the HIV clinic, participants were deemed low income since $48.6 .5 \%(n=180)$ were unemployed while those employed earned an average monthly income of 2,000-4,000 Kenya shillings $(19.7 \%, n=73)$ which is the equivalent of US \$24-\$50 (Table 1).

\section{HIV-related characteristics}

All the study participants were HIV positive and living with the infection for a median time of 3 years. With regard to the method of HIV transmission, the majority of females $(56.5 \%, n=126 p<0.05)$ and males $(48.3 \%, n=69 p<0.05)$ reported acquiring the infection from heterosexual intercourse. Regarding knowledge of a sexual partner's serostatus, $31.4 \%$ $(n=116)$ of the participants indicated their primary sex partner was living with HIV. Majority of the participants $(62.4 \%$, $n=231$ ) reported they were experiencing optimal health.

Pertaining to disclosure of serostatus to one other person(s), most females $(46.2 \%, n=103)$ had disclosed their HIV serostatus to their immediate family including brother(s), sister(s), and parent(s) while a majority of males $(65.0 \%, n=93)$ had disclosed to their spouses (Table 2).

\section{Sexual behaviors}

When asked to report condom usage the previous time they had sex, more men reported higher rates of condom use compared to women (90 versus $85 ; p<0.000)$. With regard to reporting the number of sexual partners over a 3-month period, majority of females $(45.3 \%, n=101)$ and males $(63.6 \%$, $n=91$ ) reported having sexual relations with only one partner whereas $33.2 \%(n=123)$ participants reported no sexual relations.

\section{Medication adherence}

Overall, most of the participants reported taking their prescribed HIV medication. Majority reported they had not forgotten to take their medication $(73.0 \%, n=197)$, were never careless at times to take their medication $(83.0 \%, n=224)$, never stopped taking their medication even when they felt better $(94.4 \%, n=255)$ and never stopped taking their medication even when they felt worse $(94.2 \%, n=258$; Table 3$)$. This measure also revealed a satisfactory level of internal consistency $(\alpha=0.626)$.

\section{Overall health}

When asked to report on their overall health, $62.4 \%$ $(n=231)$ of the participants deemed their health at an optimal level. 
Table 1. Participant Characteristics by Gender

\begin{tabular}{|c|c|c|c|c|c|}
\hline \multirow[b]{2}{*}{ Variable } & \multicolumn{2}{|c|}{ Female (226) } & \multicolumn{2}{|c|}{ Male (144) } & \\
\hline & Mean & $S D$ & Mean & $S D$ & \\
\hline Age (19-60) & 37.06 & 8.61 & 41.54 & 7.86 & \\
\hline \multirow[t]{2}{*}{ Years living with HIV } & 4.10 & 3.76 & 3.47 & 2.83 & \\
\hline & $\mathrm{n}$ & $\%$ & $\mathrm{n}$ & $\%$ & $\mathrm{p}<0.05$ \\
\hline Education & & & & & $0.008^{*}$ \\
\hline None/didn't attend school & 21 & 9.3 & 4 & 2.8 & \\
\hline Standard $1-3$ & 9 & 4.0 & 3 & 2.1 & \\
\hline Standard $4-8$ & 116 & 51.3 & 62 & 43.4 & \\
\hline Form 1-2 & 28 & 12.4 & 16 & 11.2 & \\
\hline Form 3-4 & 41 & 18.1 & 49 & 34.3 & \\
\hline Technical school & 2 & 0.9 & 4 & 2.8 & \\
\hline University & 4 & 1.8 & 2 & 1.4 & \\
\hline Other & 2 & 0.9 & 2 & 1.4 & \\
\hline Tribe group & & & & & 0.251 \\
\hline Luhya & 68 & 30.1 & 34 & 23.8 & \\
\hline Luo & 55 & 24.3 & 46 & 32.2 & \\
\hline Kamba & 29 & 12.8 & 16 & 11.2 & \\
\hline Bukusu & 21 & 9.3 & 10 & 7.0 & \\
\hline Kikuyu & 14 & 6.2 & 14 & 9.8 & \\
\hline Kisii & 14 & 6.2 & 5 & 3.5 & \\
\hline Relationship & & & & & $0.000^{*}$ \\
\hline Married & 78 & 34.5 & 111 & 77.6 & \\
\hline Divorced & 39 & 17.3 & 8 & 5.6 & \\
\hline Single & 33 & 14.6 & 9 & 6.3 & \\
\hline Significant other/partner & 17 & 7.5 & 4 & 2.8 & \\
\hline Widow/er & 54 & 23.9 & 9 & 6.3 & \\
\hline Raise children & & & & & 0.196 \\
\hline Yes & 198 & 87.6 & 126 & 88.1 & \\
\hline No & 12 & 5.3 & 6 & 4.2 & \\
\hline Have no children & 12 & 5.3 & 4 & 2.8 & \\
\hline Identify with religion & & & & & 0.175 \\
\hline Yes & 216 & 95.6 & 136 & 95.1 & \\
\hline No & 6 & 2.7 & 7 & 4.9 & \\
\hline Employment & & & & & $0.028^{*}$ \\
\hline No & 124 & 54.9 & 56 & 39.2 & \\
\hline Yes & 58 & 25.7 & 52 & 36.4 & \\
\hline Other & 31 & 13.7 & 21 & 14.7 & \\
\hline Average earnings & & & & & $0.000^{*}$ \\
\hline Less than ksh 1,999 & 16 & 7.1 & 7 & 4.9 & \\
\hline ksh $2,000-4,999$ & 53 & 23.5 & 20 & 14.0 & \\
\hline ksh $5,000-10,999$ & 9 & 4.0 & 31 & 21.7 & \\
\hline ksh $11,000-20,999$ & 4 & 1.8 & 10 & 7.0 & \\
\hline ksh $21,000-30,999$ & 2 & 0.9 & 5 & 3.5 & \\
\hline ksh 31,000 and above & 2 & 0.9 & 2 & 1.4 & \\
\hline Education & & & USA & Syste & ent \\
\hline Standard 1-3 & & & Elen & ool (up & de) \\
\hline Standard $4-8$ & & & Mid & up to & \\
\hline Form 1-2 & & & Low & h scho & phomore level) \\
\hline Form 3-4 & & & Higl & gh sch & enior level) \\
\hline Technical school & & & Com & lege & \\
\hline University & & & Four & ersity & \\
\hline Average earnings/month & & & USA & Equiv & \\
\hline Less than ksh 1,999 & & & Less & & \\
\hline ksh 2000-4,999 & & & $\$ 24$ & & \\
\hline ksh 5,000-10,999 & & & $\$ 50$ & & \\
\hline ksh 11,000-20,999 & & & $\$ 130$ & & \\
\hline ksh 21,000-30,999 & & & $\$ 250$ & & \\
\hline ksh 31,000 and above & & & $\$ 370$ & & \\
\hline
\end{tabular}


Table 2. Disclosure Patterns by Gender

\begin{tabular}{lrrrrrr}
\hline & \multicolumn{2}{c}{ Female } & & \multicolumn{2}{c}{ Male } & \\
\cline { 2 - 3 } & \multicolumn{1}{c}{$\mathrm{n}$} & $\%$ & & $\mathrm{n}$ & $\%$ & $\mathrm{p}^{\mathrm{a}}$ \\
\hline Anyone & 169 & 75.8 & & 110 & 76.9 & 0.860 \\
Spouse & 71 & 31.8 & & 93 & 65.0 & 0.203 \\
Children & 49 & 22.0 & & 22 & 15.4 & $\mathbf{0 . 0 1 7}^{\mathrm{a}}$ \\
Immediate family & 103 & 46.2 & & 43 & 30.1 & $\mathbf{0 . 0 4 6}^{\mathrm{a}}$ \\
Friend & 59 & 26.5 & & 30 & 21.0 & 0.071 \\
Employer & 13 & 5.8 & & 7 & 4.9 & 0.242 \\
Elder & 12 & 5.4 & & 7 & 4.9 & \\
Uncle/aunt & 27 & 12.1 & & 18 & 12.6 & \\
People at church & 16 & 7.2 & & 8 & 5.6 & \\
Grandparent & 7 & 3.1 & & 7 & 4.9 & \\
Neighbor & 25 & 11.2 & & 12 & 8.4 & \\
\hline
\end{tabular}

${ }^{\mathrm{a}} p<0.05$

\section{HIV felt stigma and depression}

Only a fair proportion of the participants reported experiencing high levels of stigma $(25.9 \%, n=96)$ compared to $73.5 \%$ $(n=272)$ who reported experiencing low levels of HIV felt stigma. Similarly, only a few participants reported severe depression $(14.3 \%, n=53)$ in comparison to $84.9 \% \quad(n=314)$ who reported mild depression.

\section{Impact of HIV felt stigma mediated by depression on HIV prevention behaviors}

Using the criterion $\alpha=0.05$, depression and personal life disrupted (factor 4) significantly predicted an impact on overall health; ostracize (factor 2) significantly predicted an impact on disclosure of serostatus; and public attitudes (factor 1) and personal life disrupted (factor 4) significantly predicted an impact on medication adherence (Table 4).

With regard to overall health, participants who reported experiencing depressive symptoms were more likely, than those not experiencing those symptoms, to have poor health (odds ratio $[\mathrm{OR}]=6.234 ; 95 \%$ confidence interval $[\mathrm{CI}]: 2.95$, $13.16, p<0.05)$, controlling for all other variables in the model. Participants experiencing felt stigma related to a disruption of their personal lives were also more likely, than those not experiencing that kind of felt stigma, to have poor health $(\mathrm{OR}=1.779$; 95\% CI: 1.03, 3.06, $p<0.05)$, controlling for all other variables in the model (Table 4 ).

Where disclosure of serostatus to one other person is concerned, participants who indicated experiencing felt stigma related to being ostracized we more likely, than those not experiencing those symptoms, to not disclose their serostatus to one other person $(\mathrm{OR}=2.139 ; 95 \% \mathrm{CI}: 1.02,4.48 ; p<0.05)$, controlling for all other variables in the model (Table 4 ).
With regard to medication adherence, participants experiencing felt stigma related to public attitudes were more likely, than those not experiencing that kind of felt stigma, to not adhere to prescribed medication regimen $(\mathrm{OR}=2.341 ; 95 \% \mathrm{CI}$ : $1.24,4.40, p<0.05)$, controlling for all other variables in the model. Participants experiencing felt stigma related to a disruption of their personal lives were also more likely, than those not experiencing that kind of felt stigma, to not adhere to prescribed medication regimen $(\mathrm{OR}=2.125 ; 95 \% \mathrm{CI}: 1.22$, $3.68, p<0.05)$, controlling for all other variables in the model (Table 4).

\section{Discussion}

The purpose of the study was to examine the extent to which HIV-related felt stigma would impact overall health and targeted HIV prevention behaviors (condom use, disclosure of HIV serostatus and adherence to prescribed HIV medication) among PLWHA in an urban city in Kenya. The support of the psychometric assessment of the felt stigma measure suggests that the instrument could have predictive capacity in examining the relationship between stigma and HIV prevention behavioral outcomes.

Findings indicate that the fair proportion of participants who reported experiencing felt stigma related to other people's attitudes towards their condition, ostracizing and a disruption of their personal life, were likely to not adhere to prescribed HIV medication and not disclose their HIV serostatus to one other person. These findings support documented evidence which highlights that stigma significantly impacts PLWHA because it may (1) interfere with disclosure of serostatus, e.g., disclosure may affect their relationships with family, friends, coworkers or (2) interferes with their seeking HIV / AIDS care services, e.g., not adhere to their medication regimen to mitigate side effects such as weight loss; and (3) can lead to an increase in HIV transmission, e.g., PLWHA on antiretroviral medication may not exhibit physical symptoms resulting in their engaging in risky sexual behaviors such as PLWHA avoiding using condoms to prevent detection of serostatus. $^{12,18,19,45}$

Additionally, those who experienced felt stigma related to a disruption of their personal lives and mediated by depressive symptoms, were likely to have poor overall health. Research reports that when a stigmatized individual perceives negative actions by other individuals as legitimate, their mental health could be affected, ${ }^{46}$ e.g., through depressive symptoms that could possibly have a negative impact on their overall health. Depression may increase PLWHA perception of HIV stigma especially when such an individual suffers from psychological issues likely to interfere with their perception of self. ${ }^{36-38}$

Table 3. HIV Medication Adherence

\begin{tabular}{|c|c|c|c|c|c|}
\hline Variable $(\mathrm{n}=270)$ & $\begin{array}{l}\text { Never }=0 \\
\mathrm{n}(\%)\end{array}$ & $\begin{array}{l}\text { Rarely }=1 \\
\mathrm{n}(\%)\end{array}$ & $\begin{array}{c}\text { Sometimes }=2 \\
\mathrm{n}(\%)\end{array}$ & $\begin{array}{l}\text { Often }=3 \\
\mathrm{n}(\%)\end{array}$ & $\begin{array}{l}\text { Always }=4 \\
\mathrm{n}(\%)\end{array}$ \\
\hline Do you ever forget to take your medications? & $197(73.0)$ & $54(20.0)$ & $9(3.3)$ & $7(2.6)$ & 0 \\
\hline Are you careless at times about taking your medications? & $224(83.0)$ & $32(11.9)$ & $11(4.1)$ & $1(0.4)$ & 0 \\
\hline $\begin{array}{l}\text { When you feel better, do you sometimes stop taking your } \\
\text { medications? }\end{array}$ & $255(94.4)$ & $10(3.7)$ & $3(1.1)$ & $1(0.4)$ & 0 \\
\hline $\begin{array}{l}\text { Sometimes if you feel worse when you take your medications, } \\
\text { do you stop taking them? }\end{array}$ & $254(94.1)$ & $7(2.6)$ & $7(2.6)$ & $1(0.4)$ & 0 \\
\hline
\end{tabular}


KINGORI ET AL.

Table 4. HIV Felt Stigma Impact on Behavioral Outcomes

\begin{tabular}{|c|c|c|c|c|c|c|}
\hline Variable & Nagelkerke $R^{2}$ & $B$ & $S E$ & Significance $^{\mathrm{a}}$ & Odds ratio & $95 \% \mathrm{CI}$ \\
\hline DV: Overall health (poor) & 0.201 & & & & & \\
\hline Public attitudes & & 0.569 & 0.310 & 0.067 & 1.767 & $0.96,3.24$ \\
\hline Ostracize & & 0.128 & 0.294 & 0.662 & 1.137 & $0.63,1.86$ \\
\hline Discrimination & & 0.168 & 0.429 & 0.695 & 1.183 & $0.51,2.42$ \\
\hline Personal life disrupted & & 0.576 & 0.278 & $0.038^{\mathrm{a}}$ & 1.779 & $1.03,3.22$ \\
\hline Depression & & 1.830 & 0.381 & $0.001^{\mathrm{a}}$ & 6.234 & $2.95,13.16$ \\
\hline DV: Disclose serostatus (not disclose) & 0.038 & & & & & \\
\hline Public attitudes & & 0.225 & 0.386 & 0.560 & 1.252 & $0.58,2.66$ \\
\hline Ostracize & & 0.761 & 0.378 & $0.044^{\mathrm{a}}$ & 2.139 & $1.02,4.48$ \\
\hline Discrimination & & -0.257 & 0.589 & 0.662 & 0.773 & $0.24,2.45$ \\
\hline Personal life disrupted & & -0.643 & 0.360 & 0.074 & 0.525 & $0.25,1.06$ \\
\hline Depression & & 0.064 & 0.464 & 0.890 & 1.066 & $0.42,2.65$ \\
\hline DV: Condom use (not use condom) & 0.037 & & & & & \\
\hline Public attitudes & & -0.345 & 0.285 & 0.226 & 0.708 & $0.40,1.23$ \\
\hline Ostracize & & -0.047 & 0.304 & 0.877 & 0.954 & $0.52,1.73$ \\
\hline Discrimination & & 0.716 & 0.458 & 0.118 & 2.046 & $0.83,5.02$ \\
\hline Personal life disrupted & & -0.466 & 0.283 & 0.100 & 0.627 & $0.36,1.09$ \\
\hline Depression & & 0.387 & 0.369 & 0.293 & 1.473 & $0.71,3.03$ \\
\hline DV: Medication adherence (not adhere) & 0.149 & & & & & \\
\hline Public attitudes & & 0.850 & 0.323 & $0.008^{\mathrm{a}}$ & 2.341 & $1.24,4.40$ \\
\hline Ostracize & & 0.083 & 0.294 & 0.778 & 1.086 & $0.61,1.93$ \\
\hline Discrimination & & 0.500 & 0.439 & 0.255 & 1.649 & $0.69,3.90$ \\
\hline Personal life disrupted & & 0.754 & 0.281 & $0.007^{\mathrm{a}}$ & 2.125 & $1.22,3.68$ \\
\hline Depression & & 0.519 & 0.361 & 0.151 & 1.681 & $0.82,3.41$ \\
\hline
\end{tabular}

${ }^{\mathrm{a}} p<0.05$

With regard to the targeted behavioral outcomes, majority of the participants reported disclosing their serostatus to one other person and taking their prescribed HIV medication. Participant disclosure patterns suggest that participants disclosed to those with whom they were comfortable. Research reports that disclosure of serostatus is selective among PLWHA given the need for social support, to prevent HV transmission to sexual partners and fear that disclosure can interfere with varied things such as employment opportunities, marriage plans, ability to have children with a spouse or confidentiality. ${ }^{18,47,48}$

To minimize the spread of HIV infection, disclosure of serostatus to sexual partners is critical. In this study, disclosure patterns show that more male participants disclosed their status to their spouses unlike female participants. These findings are similar to a study that examined disclosure reasons for HIV positive individuals. That study found that a prevailing reason for nondisclosure to sexual partners among females was "fear of abandonment." ${ }^{\text {49(p679) }}$ Such findings are concerning and HIV interventions can focus on empowering women, e.g., by training them in condom negotiation skills, to enhance disclosure and minimize HIV transmission to spouses if we assume they are also their sexual partners.

In terms of medication adherence, majority of the participants did report taking their medication as prescribed. This finding is encouraging given that access to antiretroviral medication is reported to have increased in sub-Saharan Africa $^{50}$ and contributed to a reduction in HIV / AIDS related morbidity and mortality across the globe. ${ }^{51}$ However, we need to be cautious when interpreting these findings given the self-reported nature of the data and that the medication adherence information provided by the participants was not cross-checked with patient records.
With regard to condom use, approximately one quarter of the sample reported use of condoms during sexual relations. More males reported using condoms compared to females. The low response rate related to condom use can be attributed to: (1) stigma feelings brought on by the fear of rejection from a sexual partner; (2) the sensitive nature of the information solicited or; (3) that maybe only a few participants were engaging in sexual relations. Nevertheless, study findings support the need for HIV clinics and interventions to develop relevant HIV prevention strategies targeting female PLWHA to enhance their condom negotiation skills and serostatus disclosure to their sexual partners. On another hand, most of the participants who were having sexual relations reported engaging with only one partner. Such findings are encouraging given the documented evidence that having multiple partners increases the likelihood of spreading HIV infection. Future research may need to speculate whether social desirability had any role to play in the participants reporting engaging in sexual relations with only one partner.

Although the sample is highly selective given that participants were recruited from an HIV clinic where they had been exposed to social support groups and counseling, having only a fair proportion of participants reporting experiencing HIV felt stigma is a step in the right direction. The data does not allow us to definitively assert that social support groups and counseling resulted in the low levels of HIV felt stigma. However, social support in the form of faith, friendships, family and so forth, is documented to be a buffer for stigma among those who are reportedly stigmatized. ${ }^{13,46}$ Social support is necessary given that there is a likely increase in psychological distress as HIV infection progresses. ${ }^{31}$ Future research can compare these findings using a sample of PLWHA 
who are not part of an organized HIV care system such as an HIV clinic, and find out if there would be variability.

While only a fair proportion of participants in the targeted urban sample reported feelings of stigmatization brought on by their HIV infection, it does not negate the importance of aggressively addressing HIV stigma in this population in Kenya. In fact, these findings provide an avenue for HIV interventions vis-à-vis clinics serving PLWHA, to develop focused HIV stigma prevention strategies targeting those PLWHA who have not yet been able to overcome felt stigma in Kenya albeit the 2008 HIV stigma strategic plan ${ }^{5}$ already in place. Particular attention should be given to the HIV felt stigma dimensions that emerged as significantly impacting the study's outcomes.

\section{Limitations}

Study findings may only be generalized to individuals living with HIV/AIDS in the targeted urban city in Kenya and attending an HIV clinic. Second, it is necessary to recognize that the instrument was researcher administered that may have influenced socially desirable responses. Third, from a cultural context, some of the items on the measure related to sexual activity may have been inappropriate for an older person to share with an individual young enough to be his or her daughter, which could have impacted candid responses. Fourth, even though most participants reported that they acquired HIV infection through heterosexual contact, it may be necessary to exercise caution in interpreting these findings given the limitations associated with self-reported data. Social desirability could have influenced the responses which may minimize accurate count of those who may have acquired HIV via injection drug use or homosexuality given that both activities are a crime in Kenya. Finally, although the HIV stigma instrument was deemed culturally relevant based on the psychometric evaluation, it is possible that the contextual items may not be relevant to all cultures in Kenya given that there are more than 40 tribes each with its own unique cultural context.

\section{Conclusion}

Study findings are useful for HIV clinics and interventions to provide strategies for PLWHA experiencing felt stigma in order to enhance the uptake of HIV prevention behaviors. Specifically, a concentrated effort should address dimensions of HIV felt stigma that emerged as having an impact on medication adherence, disclosure of serostatus to one other person and overall health. In order to address the emerging dimensions of HIV felt stigma effectively, policies in place such as those that identify high-risk populations from the general population may need to be revised since they may be enhancing stigma and discrimination against PLWHA. Such revisions may help enhance medication adherence, condom use and disclosure of serostatus such that those who are HIV positive will not be afraid to reveal their serostatus and thus prevent further HIV transmission to their sexual partners and have access to social support from friends, families and the community.

\section{Acknowledgments}

We graciously acknowledge the collaborative effort with AMREF. Funding for this investigation was supported by the School of Health, Physical Education, and Recreation, Department of Applied Health Science Indiana University.
Furthermore, we thank the study participants, medical officers in Kenya, AMREF, statistical consultants and research assistants. Special thanks are extended to Walter Kibet, Winnie Nzioka, Wairimu Njoroge, Yvonne Machira, Dr. Festus Ilako, Dorcas Kinuthia, Alex Thuo, Brian Blevins, Derrel Powers, Stephanie Dickinson, Dr. Michael Reece, Dr. Maresa Murray, Dr. Samuel Obeng, Dr. Enbal Shacham, Dr. Brian Dodge, Dr. Emannuel Akach, Dr. Peter Ngatia, Dr. David Ojakaa. Contents are solely the responsibility of the authors and do not necessarily represent any official view.

\section{Author Disclosure Statement}

No competing financial interests exist.

\section{References}

1. World Health Organization. Global HIV/AIDS response. Epidemic update and health sector progress towards Universal Access. Progress Report 2011. www.who.org (Last accessed September 4, 2012).

2. United Nations AIDS. Global Report. Fact Sheet. Sub-Saharan Africa. UNAIDS, Geneva, 2010. www.unaids.org (Last accessed September 3, 2012).

3. Joint United Nations Programme on HIV/AIDS (UNAIDS). How to get to zero: Faster. Smarter. Better. UNAIDS World AIDS Day Report. 2011. www.unaids.org (Last accessed September 4, 2012).

4. National AIDS, STI Control Programme, Ministry of Health, Kenya. Kenya AIDS Indicator Survey 2007: Preliminary report. Nairobi, Kenya: 2008.

5. Kenya National AIDS Control Council. Kenya national AIDS strategic plan 2009/10-2012/13. Delivering on Universal Access to Services. Nairobi, Kenya: 2009.

6. Kenya National AIDS Control Council. KENYA HIV Prevention Response and Modes of Transmission Analysis. Final Report. Nairobi, Kenya: 2009.

7. Dovidio JF, Major B, Crocker J. Stigma: Introduction and overview. In: Heatherton TF, Kleck RE, Hebl MR, Hull JG, eds. The Social Psychology of Stigma. New York: The Guilford Press, 2000;1-28.

8. Link BC, Phelan JC. Conceptualizing stigma. Annu Rev Sociol 2001;27:363-385.

9. Crocker J, Major B. Social stigma and self-esteem: The selfprotective properties of stigma. Psychol Rev 1989;96:608-630.

10. Major B, O'Brient LT. The social psychology of stigma. Annu Rev Psychol 2005;56:393-421.

11. Goffman E. Stigma: Notes on the Management of Spoiled Identity. New York: Simon \& Schuster, 1963.

12. Herek GM, Glunt EK. An epidemic of stigma: Public reactions to AIDS. Am Psychol 1988;43:886-891.

13. Buseh AG, Stevens PE. Constrained but not determined by stigma: Resistance by African American Women living with HIV. Women Health 2007;44:3.

14. Parker R, Aggleton P, Attawell K, Pulerwitz J, Brown L. HIV/AIDS-Related Stigma and Discrimination: A Conceptual Framework and an Agenda for Action. New York: Horizons Program. The Populations Council Inc., 2002.

15. Miles MS, Holditch-Davis D, Eron J, Black BP, Pedersen C, Harris DA. An HIV self-care symptom management intervention for African American mothers. Nurs Res 2003;52:350-360.

16. Bogart LM, Cowgill BO, Kennedy D, Ryan G, Murphy DA, Elijah J, Schuster MA. HIV-related stigma among people with HIV and their families: A qualitative analysis. AIDS Behav 2007;12:244-254 
17. Alonzo AA, Reynolds NR. Stigma, HIV and AIDS: An exploration and elaboration of a stigma trajectory. Soc Sci Med 1995;41:303-315.

18. Merten S, Kenter E, McKenzie O, Musheke M, Ntalasha H, Martin-Hilber A. Patient-reported barriers and drivers of adherence to antiretrovirals in sub-Saharan Africa: A metaethnography. Trop Med Int Health 2010;15:16-33.

19. Roura M, Urassa M, Busza J, Mbata D, Wringe A, Zaba B. Scaling up stigma? The effects of antiretroviral roll-out on stigma and testing. Early evidence from rural Tanzania. Sex Transm Infect 2009:308-312.

20. Airhihenbuwa C, Okoror T, Shefer T, et al. Stigma, culture, and HIV and AIDS in the western Cape, South Africa: An application of the PEN-3 cultural model for communitybased research. J Black Psychol 2009;35:407-433.

21. Simmel G. Conflict and the Web of Group Affiliations. New York: Free Press, 1955.

22. Airhihenbuwa CO. 2007 SOPHE Presidential address: On being comfortable with being uncomfortable. Centering an Africanist vision in our gateway to global health. Health Educ Behav 2007;34:31-42.

23. Ehiri JE, Anyanwu EC, Donath E, Kanu I, Jolly PE. AIDSrelated stigma in sub-Saharan Africa: Its contexts and potential intervention strategies. AIDS Public Policy J 2005;20:25-39.

24. Pescosolido BA. Beyond rational choice: The social dynamics of how people seek help. AJS 1992;97:1096-1138.

25. Jimeneza JC, Puiga M, Ramosa JC, et al. Measuring HIV felt stigma: A culturally adapted scale targeting PLWHA in Puerto Rico. AIDS Care 2010;22:1314-1322.

26. Zometa CS, Dedrick R, Knox MD, Westhoff W, Siri RS, Debaldo A. Translation, cross-cultural adaptation and validation of an HIV/AIDS knowledge and attitudinal instrument. AIDS Educ Prev 2007;19:231-244.

27. Mahendra VS, Gilborn L, Bharat S, et al. Understanding and measuring AIDS-related stigma in health care settings: A developing country perspective. I Soc Aspects HIV/AIDS 2007;4:616-625.

28. Reece M. HIV-related mental health care: Factors influencing dropout among low-income, HIV-positive individuals. AIDS Care 2003;15:707-716.

29. Sowell R, Lowenstein A, Moneyham L, Demi A, Mizuno Y, Seals B. Resources, stigma and patterns of disclosure in rural women with HIV infection. Public Health Nurs 1997;14:302-312.

30. Morisky DE, Green LW, Levine DM. Concurrent and predictive validity of a self-reported measure of medication adherence. Med Care 1986;24:67-74.

31. Reece M, Shacham E, Monahan P, et al. Psychological distress symptoms presented by individuals seeking HIVrelated psychosocial support in Western Kenya. AIDS Care 2007;19:94-1200.

32. Reece M, Basta T, Koers E. Psychological distress patterns of women accessing HIV-related mental health care. J HIV/ AIDS Soc Serv 2004;3:93-109.

33. Shacham E, Reece M, Monahan PO, et al. Measuring psychological distress symptoms in individuals living with HIV in western Kenya. J Ment Health 2008;17:424-434.

34. Spitzer RL, Kroenke K, Williams JBW. The Patient Health Questionnaire Primary Care Study Group. Validation and utility of a self-report version of PRIME-MD: The PHQ primary care study. IAMA 1999;282:1737-1744.

35. Kroenke K, Spitzer RL, Williams JBW. The PHQ-9: Validity of a brief depression severity measure. J Gen Intern Med 2001;16:606-613.
36. Wagner AC, Hart TA, Mohammed S, Ivanova E, Wong J, Loutfy MR. Correlates of HIV stigma in HIV-positive women. Arch Womens Ment Health 2010;13:207-214.

37. Wright K, Naar-King S, Lam P, Templin T, Frey M. Stigma scale revised: reliability and validity of a brief measure of stigma for HIV_Youth. J Adolesc Health 2007;40:96-98.

38. Berger BE, Ferrans CE, Lashley FR. Measuring stigma in people with HIV: Psychometric assessment of the HIV stigma scale. Res Nurs Health. 2001;24:518-529.

39. Adewuya $\mathrm{AO}, \mathrm{Ola} \mathrm{BA}, \mathrm{Afolab}$ i OO. Validity of the patient health questionnaire (PHQ-9) as a screening tool for depression amongst Nigerian university students. I Affective Disord 2006;96:89-93.

40. Moneyham L, Sowell R, Seals B, Demi A. Depressive symptoms among African American women with HIV disease. Scholarly Inquiry Nurs Pract 2000;14:9-46.

41. Perry S, Jacobsberg L, Fishman B, Weiler P, Gold J, Frances A. Psychological responses to serological testing for HIV. AIDS 1990;4:145-152.

42. Omoro SAO, Fann JR, Weymuller EA, Macharia IM, Yueh B. Swahili translation and validation of the Patient Health Questionnaire-9 depression scale in the Kenyan head and neck cancer patient population. Int J Psychiatry Med 2006;36:367-381.

43. Ware JE. SF-36 Health Survey Manual and Interpretation Guide. Boston: The Health Institute, New England Medical Center, 1996.

44. PASW (Predictive Analytics Software) Statistics version 17.0. SPSS Inc., an IBM Company Headquarters. Chicago, IL: 2010.

45. Greene S, Tucker R, Rourke SB, et al. "Under My Umbrella": The housing experiences of HIV positive parents who live with and care for their children in Ontario. Arch Womens Ment Health 2010;13:223-232.

46. Mak WWS, Kwok YTY. Internalization of stigma for parents of children with autism spectrum disorder in Hong Kong. Soc Sci Med 2010;70:2045-2051.

47. Miller M, Korves CT, Fernandez T. The social epidemiology of HIV transmission among African American women who use drugs and their social network members. AIDS Care 2007;19:858-865.

48. Green K, Faulkner SL. Expected versus actual responses to disclosure in relationships of HIV-positive African American adolescent females. Commun Stud 2002;53:297-317.

49. Ssali SN, Atuyambe L, Tumwine C, et al. Reasons for disclosure of HIV status by people living with HIV/AIDS and in HIV care in Uganda: An exploratory study. AIDS Patient Care STDs 2010;24:675-681.

50. Dahab M, Kielmann K, Charalambous S, et al. Contrasting reasons for discontinuation of antiretroviral therapy in workplace and public-sector HIV programs in South Africa. AIDS Patient Care STDs 2011;25:53-59.

51. Assefa Y, Damme WV, Mariam DH, Kloos H. Toward universal access to HIV counseling and testing and antiretroviral treatment in Ethiopia: Looking beyond HIV testing and ART initiation. AIDS Patient Care STDs 2010;24:521-525.

Address correspondence to: Caroline Kingori, Ph.D., M.P.H. Department of Social and Public Health Grover Center W347 1 Ohio University Athens OH 45701

E-mail: kingori@ohio.edu 